\title{
Clinical management of Parkinson's disease dementia: pitfalls and progress
}

\author{
Michael Samuel, Ian Maidment, Malaz Boustani \\ \& Chris Fox
}

Abstract The non-motor symptoms of Parkinson's disease, which include cognitive, behavioural and psychological problems, are significant not just in epidemiological terms but also in their impact on patients and carers. Each symptom requires careful evaluation, owing to the potential overlap with comorbid conditions and also to the multiple aetiological pathways in Parkinson's disease. Such monitoring allows enhanced management. However, the diagnostic difficulties arising from these non-motor symptoms require further research. Here we describe the efficacy and some of the problems of medication used for non-motor symptoms, including antiparkinsonian medication, antipsychotics and acetylcholinesterase inhibitors.

The symptoms of Parkinson's disease can be broadly grouped into two domains: motor and non-motor. Motor symptoms were recognised first and they are the clinical hallmarks of a diagnosis of Parkinson's disease. More recently, there has been increased recognition of the high prevalence, disabling burden and management challenges of the non-motor symptoms. These include sleep disturbance, pain, autonomic dysfunction, and cognitive, behavioural and psychological problems. Cognitive deficit is especially common.

Here we focus on the cognitive, behavioural and psychological manifestations, outlining their prevalence, phenomenology, diagnosis and impact, and options for their psychopharmaceutical treatment. It is important to note, however, that the impact of non-motor symptoms must be considered in union with the motor symptoms. This is because it is frequently the interaction of motor and nonmotor symptoms (often called the motion-emotion balance) that affects functional performance and quality of life and creates the dilemmas of balancing the management of disabling symptoms from two different spectra.

\section{Symptom domains}

\section{Cognitive deficit}

Dementia is an acquired syndrome of decline in memory and at least one other cognitive domain (e.g. language, visuospatial or executive dysfunction) that is sufficient to interfere with social and occupational function in an alert person (American Psychiatric Association, 1994). The presence of an unimpaired consciousness is required (i.e. it is not delirium). Dementia that occurs in people with Parkinson's disease is classified in DSM-IV as 'dementia due to Parkinson's disease' - the essential feature is of dementia that is a direct pathophysiological consequence of Parkinson's disease (American Psychiatric Association, 1994). However, many of the core cognitive symptoms are omitted in the DSM-IV definition and there is as yet no clear agreement whether dementia in Parkinson's disease (here we call it 'Parkinson's disease dementia') is a pathological entity distinct from dementia with Lewy bodies (Lewy-body dementia) (Aarsland et al, 2004).

\footnotetext{
Michael Samuel is a consultant neurologist and senior lecturer with East Kent Hospitals NHS Trust, King's College Hospital London and the University of Kent. He has an interest in the neuropsychiatric aspects of Parkinson's disease, has been involved in related clinical trials and runs a movement disorder service. Ian Maidment is a specialist mental health pharmacist and is involved in a tertiary service for the psychiatric aspects of Parkinson's disease. Malaz Boustani is interested in the behavioural aspects of cognitive impairment, is involved in intervention studies and has worked on one of the largest ongoing US studies on the prevention of cognitive decline. Chris Fox is a consultant psychiatrist in Canterbury (East Kent Social Care and Partnership Trust, Canterbury, Kent, England, UK. E-mail: gfox@ doctors.org.uk). He is involved in intervention studies, a tertiary service for the psychiatric aspects of Parkinson's disease and a research clinic. He is also conducting research on other dementias and has close collaboration with US groups studying screening programmes for cognitive impairment. Ian Maidment, Malaz Boustani and Chris Fox are all working on the Cochrane Systematic Review of cholinesterase inhibitors in Parkinson's disease dementia, for which Mr Maidment is lead and Professor Boustani and Dr Fox are co-reviewers.
} 
The aetiology of the cognitive impairment in Parkinson's disease is unclear. Marked deficits in central cholinergic function, exceeding those in Alzheimer's disease, are increasingly being recognised (Bohnen et al, 2005). However, the large variety of medications currently available for the management of Parkinson's disease, which include anticholinergic drugs, may contribute to at least transient cognitive deficits. Neuroleptic drugs may also exacerbate both Parkinson's disease and cognitive impairment. Moreover, people with Parkinson's disease are often prone to the development of common comorbid medical conditions such as aspiration pneumonia, urinary tract infections and constipation, and to falls. Therefore patients with new-onset cognitive dysfunction should be evaluated for delirium.

Cognitive symptoms not explicable by the above factors occur in $25-30 \%$ of people with Parkinson's disease (Aarsland et al, 2002; Nilsson, 2004). This prevalence is six times greater than that among the population in general (Aarsland et al, 2001a). People with early Lewy-body dementia and Parkinson's disease dementia have generally less severe visual and verbal memory deficits, but more marked executive dysfunction (reasoning, planning, sequencing) than people with Alzheimer's disease (Aarsland et al, 2004). Other cognitive deficits, such as dysphasia, apraxia, alexia, agraphia, anomia and acalculia, are less pronounced, although they have been noted rarely in early Parkinson's disease in the absence of dementia (Dubois \& Pillon, 1997). Parkinson's disease dementia impairs quality of life, excerbates caregivers' distress, increases the likelihood of residential care and doubles mortality (Burn \& McKeith, 2003). This is probably partly because of the additional cognitive symptoms themselves and partly because the onset of dementia restricts the use of medications for motor symptoms. Dementia therefore indirectly adds to the motor burden of Parkinson's disease by restricting treatment of motor symptoms.

\section{Behavioural and psychological symptoms Psychosis}

The psychiatric symptoms of Parkinson's disease dementia are common, complex and disabling. Their prevalence in patients attending movement disorder clinics is between 21 and $46 \%$ (Barnes \& David, 2001). Some symptoms are noteworthy because they are mild, patients may have retained insight and require no specific therapy other than reassurance. These include vivid dreams, visual illusions (e.g. interpreting a crumb as an ant) and ideas of presence (believing that someone is in the room just visible out of the corner of an eye).
However, more severe psychotic symptoms are a significant risk factor for placement in a nursing home (Aarsland et al, 2000). Age, sleep disturbances, dementia and disease severity are significantly associated with the development of these psychotic symptoms (Barnes \& David, 2001; Barnes et al, 2003; Fenélon et al, 2000). Most patients are also taking antiparkinsonian medication, which may be associated with psychotic symptoms. Hallucinations are four times more common in people with Parkinson's disease dementia than in those with Parkinson's disease alone (Aarsland et al, 2001b).

Phenomenologically, psychotic symptoms are indistinguishable between Parkinson's disease dementia and Lewy-body dementia (Klatka et al, 1996; Aarsland et al, 2001b), but the frequency of occurrence is greatest in the latter. Hallucinations are more common in both Parkinson's disease dementia and Lewy-body dementia than in Alzheimer's disease (Aarsland et al, 2001b). Hallucinations are mostly visual, but can be auditory or tactile, in which case they usually coexist with visual hallucinations (Inzelberg et al, 1998). Hallucinations commonly involve people or animals and may or may not be threatening to the patient. If they are, they are more likely to require treatment. Delusions may involve the belief of a spouse's sexual infidelity, which can be very distressing to both patient and carer.

Medication for motor disorders can have psychiatric side-effects, just as medication for psychiatric disorders can have motor side-effects. For example, psychosis may be triggered by changes in antiparkinsonian drug therapy (Nilsson, 2004), although the exact relationship with antiparkinsonian medication is unclear. Peak-dose plasma levels of levodopa do not appear to be a direct trigger (Goetz et al, 1998a). Catechol-o-methyltransferase (COMT) inhibitors prolong the duration of action of levodopa and are probably less likely to aggravate psychosis than are other antiparkinsonian drugs. Most studies of dopamine agonists (co-prescribed with levodopa) have reported hallucinations as a side-effect (Table 1 ), although there are no direct comparisons of the

Table 1 Quoted prevalence of hallucinations as a sideeffect of antiparkinsonian drugs in major studies

\begin{tabular}{|c|c|c|}
\hline Drug & $\begin{array}{c}\text { Prevalence, } \\
\%\end{array}$ & Study \\
\hline Ropinirole & 17 & Rascol et al, 2000 \\
\hline Pramipexole & 9 & $\begin{array}{l}\text { Parkinson Study } \\
\text { Group, } 2000\end{array}$ \\
\hline Cabergoline & 4.8 & Bracco et al, 2004 \\
\hline Levodopa & $3.3-5.6$ & $\begin{array}{l}\text { Parkinson Study } \\
\text { Group, 2000; } \\
\text { Bracco et al, 2004; } \\
\text { Rascol et al, 2000 }\end{array}$ \\
\hline
\end{tabular}


agonists to assess whether these apparent differences are significant. Anticholinergic drugs, amantadine and monoamine oxidase B (MAO-B) inhibitors are also implicated.

Medications that are often effective for psychotic symptoms, such as the atypical antipsychotics risperidone and olanzapine, may worsen movement disorder (Graham et al, 1998; Friedman \& Factor 2000; Factor et al, 2002; Ondo et al, 2002), in some cases to the point where they cannot be reintroduced. Newer atypicals such as aripiprazole and quetiapine may have less propensity for these side-effects (Fernandez et al, 2004b; Juncos et al, 2004), although quetiapine has also been reported to increase paradoxical stereotypical behaviour and to cause akathisia (Prueter et al, 2003; Miwa et al, 2004).

Thus, it is clear that treatment of psychosis in Parkinson's disease is currently unsatisfactory.

\section{Depression}

Depression is common in Parkinson's disease (Nilsson, 2004), with a reported prevalence of up to $40 \%$ (Cummings, 1992). Study designs and interpretation are hindered by participants' common symptoms of apathy, anxiety, low motivation and motor complaints (Pluck \& Brown, 2002). Depression is similar in Lewy-body dementia and Parkinson's disease dementia (Klatka et al, 1996; Tandberg et al, 1996), but it may differ in Parkinson's disease without dementia.

It is not clear whether depression in Parkinson's disease is similar to depression in other nonparkinsonian disorders, nor whether it is a reaction to the illness, loss of movement or independence, nor whether it is a distinct clinical condition resulting from the dopaminergic and/or non-dopaminergic neurodegeneration that occurs in Parkinson's disease (Rascol et al, 2002). A patient with Parkinson's disease should have a thorough drug review and full medical assessment once behavioural and psychological symptoms have developed.

\section{Movement disorder}

Bradykinesia, tremor, rigidity and postural imbalance are core symptoms of Parkinson's disease. There are three common scenarios in which the motionemotion balance may become clinically significant:

- as motor symptoms increase, an upward titration of antiparkinsonian medication is required to reduce parkinsonism; this can lead to a new, drug-related decline in cognition and/ or new neuropsychiatric symptoms (Fig. 1);

- with continued neurodegeneration, despite stable medication new cognitive, behavioural and psychological symptoms may develop,

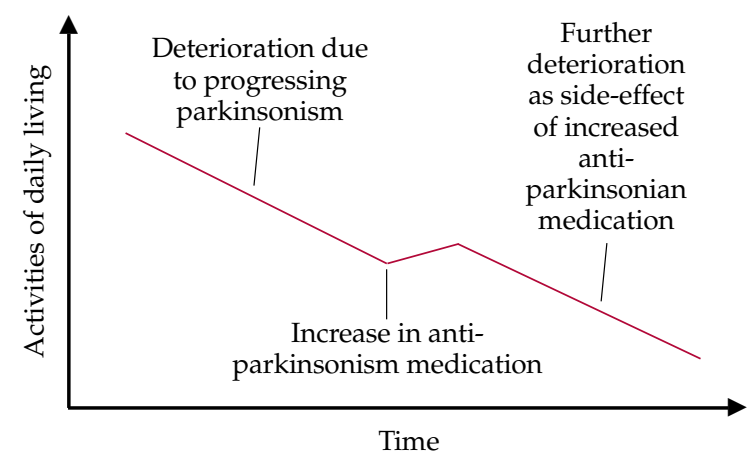

Fig. 1 Model of the possible impact of increasing antiparkinsonian medication. The neuropsychiatric sideeffects of antiparkinsonian drugs include cognitive, behavioural and psychological symptoms.

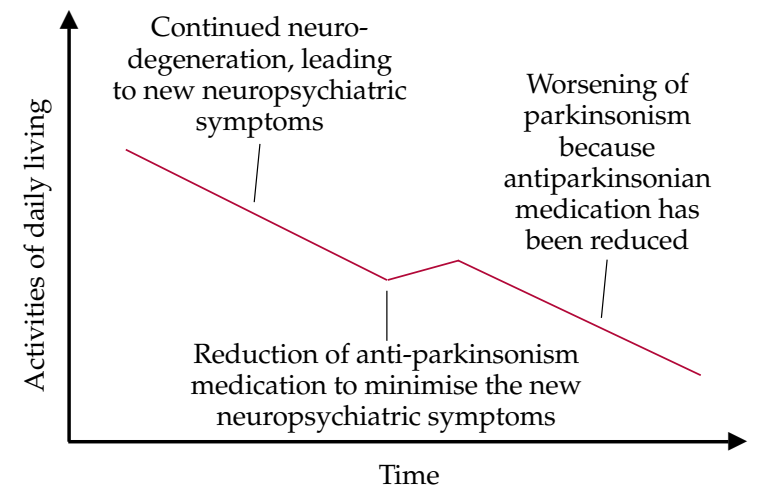

Fig. 2 Model of progressing disease possibly leading to new neuropsychiatric (cognitive, behavioural and psychological) symptoms, leading to the need for a reduction of antiparkinsonian medication.

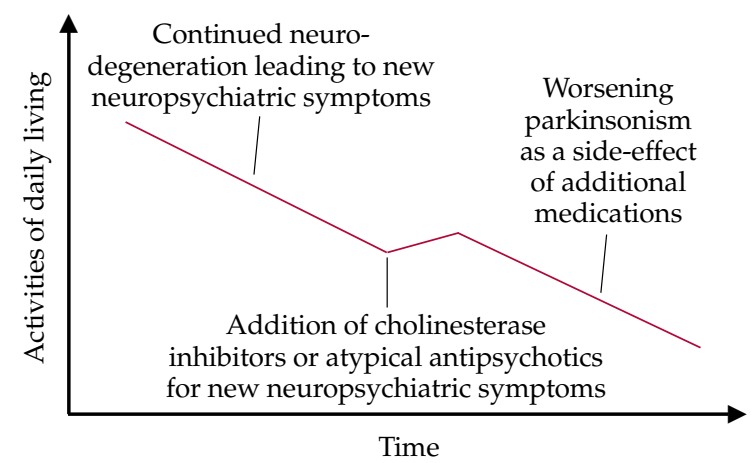

Fig. 3 Model of the possible effect on motor symptoms of additional medication for non-motor symptoms.

leading to the need for a reduction of antiparkinsonian medication (Fig. 2);

- an increase in parkinsonism can occur as a specific side-effect of additional medication required to stabilise cognitive deficits (cholinesterase inhibitors) or psychosis (atypical antipsychotics) that cannot be treated by non-pharmacological methods (Fig. 3). 


\section{Diagnostic issues}

Although the diagnosis of Parkinson's disease usually rests on well-established clinical criteria defined by the UK Parkinson's Disease Society Tissue Bank (Gibb \& Lees, 1988), there is no consensus on whether the parkinsonism of Parkinson's disease is phenotypically different from Lewy-body dementia. A diagnosis of Lewy-body dementia is potentially applicable to people with Parkinson's disease who develop dementia. If both motor and cognitive symptoms develop within 12 months it is conventional to give a diagnosis of Lewybody dementia. A diagnosis of Parkinson's disease dementia is given if the parkinsonian symptoms have existed for at least 12 months before dementia develops. These guidelines were established by an international workshop on the diagnosis of Lewybody dementia in 1996 (McKeith et al, 1996). There is no clear consensus: for example, a recent trial used a symptom period of at least 2 years, to include 'boundary' patients who had been symptomatic for periods such as 12 months and 1 week (Emre et al, 2004).

Numerous factors complicate the diagnosis of dementia in Parkinson's disease. The motor deficits may interfere with neuropsychological assessment to determine whether dementia is also present (Dubois \& Pillon, 1997). Psychotic symptoms may be induced by medication or they may be the first indication of dementia (Goetz et al, 1998b). Depressive symptoms may interfere with cognition (Brown et al, 1994), and symptoms such as increasing apathy due to dementia need to be disentangled from depression.

Structural imaging methods are being developed to facilitate differentiation of the dementias. Medial temporal lobe atrophy appears to be a useful sign for differentiating Lewy-body dementia from Alzheimer's disease (Burton et al, 2004; Samuel \& Colchester, 2005), but there is no conclusive evidence that routine structural magnetic resonance imaging (MRI) can differentiate Parkinson's disease dementia from Lewy-body dementia. Newer non-routine structural MRI techniques such as voxel-based morphometry (VBM), volumetry and diffusion tensor imaging (DTI) are being investigated as potential diagnostic tools for neurodegenerative disorders (Samuel \& Colchester, 2005). Functional imaging using positron emission tomography (PET) is not readily available in most clinical centres, but single-photon emission computed tomography (SPECT) is more accessible.

Although differentiation of Parkinson's disease dementia and Lewy-body dementia from Alzheimer's disease appears more robust, the major detectable factor for differentiating Parkinson's disease from
Lewy-body dementia using SPECT appears to be a greater caudate involvement in the latter (Colloby \& O'Brien, 2004; Walker et al, 2004). A SPECT study of blood flow showed a similar pattern of deficit in Parkinson's disease dementia and Lewy-body dementia, with reduced perfusion of the precuneus and parietal cortex, locations thought to be associated with visual processing (Firbank et al, 2003). These methods, like non-routine structural MRI, are currently considered to be at too early a stage of development for use in routine clinical practice.

\section{Therapeutic efficacy}

Several open-labelled and uncontrolled studies have been conducted using various drugs to treat non-motor symptoms of Parkinson's disease, but we will concentrate our discussion on double-blind placebo-controlled randomised clinical trials (RCTs). For brevity, we have summarised key points of each trial, rather than attempting to describe them or their results in detail.

\section{Cholinesterase inhibitors for cognitive decline}

Three RCTs have evaluated cholinesterase inhibitors for cognitive impairment in Parkinson's disease: two studied donepezil (Aarsland et al, 2002; Leroi et al, 2004) and the third, rivastigmine (Emre et al, 2004).

\section{Rivastigmine study}

The largest of the three (541 participants) is the rivastigmine study (Emre et al, 2004), which was a 24-week prospective parallel-group trial investigating the efficacy, tolerability and safety of 3-12 mg rivastigmine in the treatment of mild-tomoderate DSM-IV-compatible Parkinson's disease dementia (the diagnoses of Parkinson's disease met UK Parkinson's Disease Society Tissue Bank clinical criteria). Patients in the rivastigmine group were maintained on the maximum tolerated dose (the mean daily dose was $8.6 \mathrm{mg}$ ).

The primary outcomes were assessed using the cognitive sub-scale of the Alzheimer's Disease Assessment Scale (ADAS-Cog) and the Alzheimer's Disease Cooperative Study - Clinician's Global Impression of Change (ADCS-CGIC). Instruments used to assess secondary outcomes included the Alzheimer's Disease Cooperative Study - Activities of Daily Living, the 10-item Neuropsychiatric Inventory (NPI) and the Mini-Mental State Examination (MMSE).

Rivastigmine improved the mean ADAS-Cog score by 2.1 points compared with a 0.7 point 
deterioration with placebo $(\mathrm{P}<0.001)$. On the ADCS-CGIC, marked or moderate improvement was noted for $20 \%$ of the rivastigmine group and $15 \%$ of the placebo group, and marked or moderate worsening was recorded for $13 \%$ of the rivastigmine group and $23 \%$ of the placebo. Compared with placebo, rivastigmine produced statistically significant greater improvements on every secondary outcome.

In total, 131 participants stopped treatment: $27 \%$ of the rivastigmine group and $18 \%$ of the placebo group. The main reason for discontinuation was adverse events: $17 \%$ (rivastigmine) and $8 \%$ (placebo). The most common adverse events were nausea $(29 \%$ on rivastigmine $v .11 \%$ on placebo, $P<0.001)$, vomiting $(16 \%$ v. $2 \%, P<0.001)$ and tremor $(10 \%$ v. $4 \%, \mathrm{P}=0.01)$.

\section{Donepezil: study 1}

Aarsland et al (2002) conducted a crossover study of donepezil to treat 14 out-patients with DSM-IVcompatible Parkinson's disease dementia or probable Parkinson's disease dementia (the diagnostic criteria were not reported). The study involved two 10-week treatment periods and no washout period. Donepezil was increased from 5 to $10 \mathrm{mg}$ at 6 weeks.

Scales used included the MMSE, the NPI, the Unified Parkinson's Disease Rating Scale (UPDRS) and the Clinician's Interview Based Impression of Change, including caregiver information (CIBICplus). Donepezil improved the mean MMSE score by 2.1 points compared with a 0.3 point improvement on placebo $(P=0.013)$. The CIBIC-plus was 3 or less in $42 \%$ of the donepezil group and $17 \%$ of the placebo group, and the mean score was significantly lower with donepezil ( 3 v. 4, $P=0.034)$. Three participants had missing CIBIC-plus data and were coded as no change. When these participants were excluded this significance fell to $P<0.079$. There was no significant group difference in the UPDRS change scores.

Twelve participants finished the study; two participants taking donepezil withdrew owing to adverse events (gastrointestinal problems and dizziness).

\section{Donepezil: study 2}

The second donepezil RCT (Leroi et al, 2004) lasted 18 weeks and involved 16 out-patients with DSMIV-compatible Parkinson's disease dementia or cognitive impairment secondary to Parkinson's disease (in each case Parkinson's disease was diagnosed using UK Parkinson's Disease Society Tissue Bank clinical criteria). Donepezil was started at $2.5 \mathrm{mg}$ daily and increased to $10 \mathrm{mg}$ daily over 7 weeks. The mean age was 66 years for the donepezil group and 70 years for the placebo group.
The scales used included the Dementia Rating Scale (DRS), MMSE, NPI and UPDRS. Significantly higher mean daily doses of placebo compared with donepezil were tolerated $(8.9$ v. $6.4 \mathrm{mg}$, $P=0.03)$. Participants taking the placebo remained in the study significantly longer than those taking donepezil (17 v. 13 weeks, $P<0.05)$. The results were reported as $\delta$-scores (donepezil change score minus placebo change score), with positive scores favouring donepezil and negative scores favouring placebo. The DRS total $\delta$-score was -0.7 (non-significant) and the DRS memory $\delta$-subscore was $3.3(P=0.03)$. The $\delta$-score for the MMSE $(-1.1)$ was non-significant. The UPDRS $\delta$-scores for motor symptoms, activities of daily living and complications of therapy were 1.65, 0.83 and -0.22 respectively, all non-significant.

Discontinuation owing to adverse events was more frequent with donepezil (71\% v. $11 \%, P$ not stated). Side-effects leading to donepezil discontinuation included diplopia, lightheadedness, constipation, nausea/vomiting, hypersalivation, frequent urination, motor disturbances and rhinorrhea.

\section{Summary}

Although cholinesterase inhibitors appear to have a moderately beneficial effect on cognitive deficits in Parkinson's disease dementia, there is a relatively high drop-out rate owing to adverse reactions (rivastigmine $17 \%$, donepezil up to $71 \%$ ) and patients started on these agents should be monitored carefully for non-specific side-effects or deterioration of parkinsonism, especially tremor (Maidment et al, 2006).

\section{Cholinesterase inhibitors for hallucinations}

There is a rationale for trying to treat hallucinations in Parkinson's disease dementia and Lewy-body dementia with cholinesterase inhibitors, which may also be beneficial for the cognitive decline itself.

The effect of cholinesterase inhibitors on psychosis has not been studied specifically in Parkinson's disease dementia and the data given here have been gathered from either small open-labelled case series of people with Parkinson's disease or secondary outcomes from the RCTs for allied disorders. In the RCTs neuropsychiatric symptoms, including hallucinations, were rated with the NPI.

\section{Donepezil in Parkinson's disease}

In a small open series, donepezil reduced visual hallucinations without worsening motor dysfunctions in three individuals with Parkinson's 
disease, but caused delusions in one of the three (Kurita et al, 2003). Another small 2-month study examining the effect of donepezil on both hallucinations and delusions in Parkinson's disease showed a significant improvement in both at the expense of deterioration in motor symptoms in two of the eight participants (Fabbrini et al, 2002).

In one small-scale donepezil RCT (Aarsland et al, 2002), the baseline NPI scores were extremely low and the changes too insignificant to merit reporting. In the second small-scale donepezil RCT (Leroi et al, 2004), donepezil had no significant effect on NPI scores.

\section{Rivastigmine in Parkinson's disease}

In the largest of the three RCTs, that by Emre et al (2004), rivastigmine produced a significant improvement in NPI scores. However, it is not clear whether the improvement of 2.0 relative to placebo on the NPI (scored from 0 to 120) is clinically significant. Baseline data indicate that floor effects may have affected the results, and individual NPI item scores from the trial have yet to be published. The observation that hallucinations were experienced by $10 \%$ of patients taking placebo and $5 \%$ of patients taking rivastigmine $(P=0.04)$ suggests mild benefit.

\section{Comparison results for Lewy-body dementia}

Hallucinations are more common in Lewy-body dementia than in Parkinson's disease dementia. An intention-to-treat analysis of an RCT involving 120 people with Lewy-body dementia showed that $50 \%$ of those receiving rivastigmine achieved a $>30 \%$ improvement in a grouped cluster of psychiatric symptoms (hallucinations, delusions, apathy and depression), compared with $30 \%$ of those on placebo. The incidence of cholinergic side-effects was slightly higher in the rivastigmine group, and three patients on rivastigmine became severely agitated (McKeith et al, 2000). According to the UPDRS scores, there was no worsening of parkinsonism; tremor, however, was more frequent with rivastigmine. The study also excluded individuals with severe parkinsonism so an extrapolation to Parkinson's disease dementia (in which co-existing parkinsonism is likely to be more severe and hallucinations less frequent) may be premature.

\section{Summary}

Although there is preliminary evidence that cholinesterase inhibitors reduce hallucinations in Parkinson's disease without dementia, there are no other clear data on their efficacy in the management of severe psychosis in such patients (Poewe, 2003).

\section{Atypical antipsychotics for psychosis}

Risperidone and olanzapine

Although small-scale studies have indicated that hallucinations and delusions are reduced by risperidone and olanzapine, a significant deterioration in motor function, leading to high drop-out rates, occurred in more controlled studies (Ellis et al, 2000; Friedman \& Factor, 2000; Goetz et al, 2000; Breier et al, 2002; Factor et al, 2002; Ondo et al, 2002).

\section{Clozapine}

Two RCTs showed a beneficial effect of clozapine on three measures of psychosis (the Clinical Global Impression scale, Brief Psychiatric Rating Scale and the Scale of Assessment of Positive Symptoms) over 14 months (French Clozapine Parkinson Study Group, 1999; Parkinson Study Group, 1999). Despite these results, and despite the reported improvement of tremor with clozapine, its practical use in a routine hospital setting is limited because of the need for meticulous monitoring to reduce the risk of agranulocytosis. Other risks include cardiac, renal and thrombotic problems (Poewe, 2003).

\section{Quetiapine}

Placebo-controlled RCTs of the use of quetiapine in Parkinson's disease are still underway, but one comparing quetiapine and clozapine has now been published (Morgante et al, 2004). Using the same rating scales as the above-mentioned clozapine $v$. placebo trials, it reports the encouraging result that, although there was no significant difference between quetiapine and clozapine in efficacy at resolving psychosis, unlike risperidone and olanzapine they caused no deterioration in parkinsonism. The results of the quetiapine $v$. placebo trials are therefore eagerly awaited.

\section{Aripiprazole}

One newer atypical antipsychotic drug, aripiprazole, which has a partial $\mathrm{D}_{2}$ and 5- $\mathrm{HT}_{1 \mathrm{a}}$ receptor agonist action, has been evaluated in a small open-labelled trial (Fernandez et al, 2004). Efficacy was limited to benefit in only two of the eight participating patients, but the other six discontinued the drug, two because of worsening parkinsonism.

\section{Summary}

Clozapine is the most effective drug for psychosis in Parkinson's disease, but the practical limitations of its use have led to trials of other atypical antipsychotics that do not require the intensive monitoring that 
is necessary for clozapine. Of these, quetiapine shows the most promise, but placebo-controlled trials are lacking. The use of olanzapine, risperidone and aripiprazole appears limited by unacceptable motor deterioration. However, the safety of using antipsychotics in Parkinson's disease dementia needs further evaluation, as it is uncertain whether the findings from studies of Alzheimer's and other non-specific dementias apply (Ballard et al, 2005; Layton et al, 2005). Studies are underway that will address this issue.

\section{Conclusions}

Parkinson's disease dementia can be difficult to diagnosis. It is not clear whether Parkinson's disease dementia and Lewy-body dementia are distinct syndromes. Furthermore, motor deficits, depressive or other psychiatric symptoms, and cognitive impairment not amounting to dementia may all also contribute to the diagnostic problem. Medicines that treat symptoms in one domain may worsen symptoms in other domains. When assessing the efficacy of a medication it is necessary to consider the patient's global functioning and to include an assessment of activities of daily living. A treatment programme should incorporate assessments of both motor and non-motor symptoms. There are limited data on best clinical practice for combined motor, cognitive and psychiatric symptoms, but the development of cholinesterase inhibitors, newer atypical antipsychotics and large RCTs may herald significant developments for optimal management of these complex interactions. Research is needed to evaluate the tolerability of psychiatric medication as well as other medication that enhances cognition in Parkinson's disease dementia (Fox et al, 2005).

Key learning points for this article are shown in Box 1.

\section{Declaration of interest}

M.S. has received research funding from Eisai/ Lundbeck, Novartis and the Parkinson's Disease Society. I. M. has received research funding from the Parkinson's Disease Society, Eisai/Lundbeck and Novartis, and honoraria from Schwarz Pharma, Shire Pharmaceuticals Group, Lilly and Astra Zeneca. M.B. has received research funding from Pfizer Inc. C.F. has received research funding from the Parkinson's Disease Society, Shire Pharmaceuticals Group, Eisai/Lundbeck and Novartis, and honoraria from Lundbeck, Shire Pharmaceuticals Group, Lilly and Astra Zeneca. None of the authors has any shares or financial interests in pharmaceutical companies.

\section{Box 1 Key learning points}

- Consideration of the motion-emotion balance is paramount when prescribing medication in Parkinson's disease dementia

- Motor deficits, other psychiatric symptoms and 'cognitive impairment not dementia' must be considered in the assessment of Parkinson's disease dementia

- Caution should be exercised with psychotic disorders in Parkinson's disease dementia. If antipsychotics are deemed necessary, clozapine appears to be effective, but it can be difficult to use. Studies of quetiapine are underway

- Cholinesterase inhibitors appear to offer hope for Parkinson's disease dementia but tolerability should be carefully monitored and the potential for a worsening of movement symptoms, particularly tremor, should be considered.

\section{References}

Aarsland, D., Larsen, J. P., Tandberg, E., et al (2000) Predictors of nursing home placement in Parkinson's disease: a populationbased, prospective study. Journal of the American Geriatric Society, 48, 938-942.

Aarsland, D., Anderson, K., Larsen, J. P., et al (2001a) Risk of dementia in Parkinson's disease - a community-based, prospective study. Neurology, 56, 730-736.

Aarsland, D., Ballard, C., Larsen, J. P., et al (2001b) A comparative study of psychiatric symptoms in dementia with Lewy bodies and Parkinson's disease with and without dementia. International Journal of Geriatric Psychiatry, 16, 528-536.

Aarsland, D., Laake, K., Larsen, J. P., et al (2002) Donepezil for cognitive impairment in Parkinson's disease: a randomised control trial. Journal of Neurology, Neurosurgery and Psychiatry, 72, 708-712.

Aarsland, D., Ballard, C. G. \& Halliday, G. (2004) Are Parkinson's disease with dementia and dementia with Lewy bodies the same entity? Journal of Geriatric Psychiatry and Neurology, 17, 137-145.

American Psychiatric Association (1994) Diagnostic and Statistical Manual of Mental Disorders (DSM-IV) (4th edn). Washington, DC: APA.

Ballard, C., Margello-Lana, M., Juszczak, E., et al (2005) Quetiapine and rivastigmine and cognitive decline in Alzheimer's disease: randomized double blind placebo controlled trial. $B M J, 330$, 857-858.

Barnes, J. \& David, A. S. (2001) Visual hallucinations in Parkinson's disease: a review and phenomenological survey. Journal of Neurology, Neurosurgery and Psychiatry, 70, 727-733.

Barnes, J., Boubert, L., Harris, J., et al (2003) Reality monitoring and visual hallucinations in Parkinson's disease. Neuropsychologia, 41, 565-574.

Bohnen, N. I., Kaufer, D. I., Hendrickson, R., et al (2005) Cognitive correlates of cortical cholinergic denervation in Parkinson's disease and parkinsonian dementia. Journal of Neurology, 252, 9, doi: 10.1007/s00415-005-0971-0

Bracco, F., Battaglia, A., Chouza, C., et al (2004) The long-acting dopamine receptor agonist cabergoline in early Parkinson's disease: final results of a 5-year, double-blind, levodopacontrolled study. CNS Drugs, 18, 733-746.

Breier, A., Sutton, V. K., Feldman, P. D., et al (2002) Olanzapine in the treatment of dopamimetic-induced psychosis in patients with Parkinson's disease. Biological Psychiatry, 52, 438-445. 
Brown, R. G., Scott, L. C., Bench, C. J., et al (1994) Cognitive function in depression: its relationship to the presence and severity of intellectual decline. Psychological Medicine, 24, 829-847.

Burn, D. J. \& McKeith, I. G. (2003) Current treatment of dementia with Lewy bodies and dementia associated with Parkinson's disease. Movement Disorders, 18, S72-S79.

Burton, E. J., McKeith, I. G., Burn, D. J., et al (2004) Cerebral atrophy in Parkinson's disease with and without dementia: a comparison with Alzheimer's disease, dementia with Lewy bodies and controls. Brain, 127, 791-800.

Colloby, S. \& O'Brien, J. (2004) Functional imaging in Parkinson's disease and dementia with Lewy bodies. Journal of Geriatric Psychiatry and Neurology, 17, 158-163.

Cummings, J. L. (1992) Depression and Parkinson's disease. American Journal of Psychiatry, 149, 443-454

Dubois, B. \& Pillon, B. (1997) Cognitive deficits in Parkinson's disease. Journal of Neurology, 244, 2-8.

Ellis, T., Cudkowicz, M. E., Sexton, P. M., et al (2000) Clozapine and risperidone treatment of psychosis in Parkinson's disease. Journal of Neuropsychiatry and Clinical Neurosciences, 12, 364-369.

Emre, M., Aarsland, D., Albanese, A., et al (2004) Rivastigmine for dementia associated with Parkinson's disease. New England Journal of Medicine, 351, 2509-2518.

Fabbrini, G., Barbanti, P., Aurilia, C. P., et al (2002) Donepezil in the treatment of hallucinations and delusions in Parkinson's disease. Neurological Sciences, 23, 41-43.

Factor, S. A., Molho, E. S. \& Friedman, J. H. (2002) Risperidone and Parkinson's disease. Movement Disorders, 17, 221-222.

Fenélon, G., Mahieux, F., Huon, R., et al (2000) Hallucinations in Parkinson's disease: prevalence, phenomenology and risk factors. Brain, 123, 733-745.

Fernandez, H. H., Trieschmann, M. E. \& Friedman, J. H. (2004) Aripiprazole for drug-induced psychosis in Parkinson's disease: preliminary experience. Clinical Neuropharmacology, 27, 4-5.

Firbank, M. J., Colloby, S. J., Burn, D. J., et al (2003) Regional cerebral blood flow in Parkinson's disease with and without dementia. Neuroimage, 20, 1309-1319.

Fox, C. G., Samuel, M., Beats, B., et al (2005) Memantine in Parkinson's disease dementia: clinical experience. Movement Disorders, 20, 418.

French Clozapine Parkinson Study Group (1999) Clozapine in drug-induced psychosis in Parkinson's disease. Lancet, 353, 2041-2042

Friedman, J. H. \& Factor, S. A. (2000) Atypical antipsychotics in the treatment of drug-induced psychosis in Parkinson's disease. Movement Disorders, 15, 201-211.

Gibb, W. R. \& Lees, A. J. (1988) The relevance of the Lewy body to the pathogenesis of idiopathic Parkinson's disease. Journal of Neurology, Neurosurgery and Psychiatry, 51, 745-752.

Goetz, C. G., Pappert, E. J., Blasucci, L. M., et al (1998a) Intravenous levodopa in hallucinating Parkinson's disease patients: highdose challenge does not precipitate hallucinations. Neurology, 50, 515-517.

Goetz, C. G., Vogel, C., Tanner, C. M., et al (1998b) Early dopaminergic drug induced hallucinations in Parkinson's disease. Neurology, 51, 811-814.

Goetz, C. G., Blasucci, L. M., Leurgans, S., et al (2000) Olanzapine and clozapine: comparative effects on motor function in hallucinating PD patients. Neurology, 55, 789-794.

Graham, J. M., Sussman, J. D., Ford, K. S., et al (1998) Olanzapine in the treatment of hallucinosis in idiopathic parkinson's disease: a cautionary note. Journal of Neurology, Neurosurgery and Psychiatry, 65, 774-777.

Inzelberg, R., Kipervasser, S. \& Korczyn, A. D. (1998) Auditory hallucinations in Parkinson's disease. Journal of Neurology, Neurosurgery and Psychiatry, 64, 533-535.

Juncos, J. L., Roberts, V. J., Evatt, M. L., et al (2004) Quetiapine improves psychotic symptoms and cognition in Parkinson's disease. Movement Disorders, 19, 29-35.

Klatka, L. A., Louis, E. D. \& Schiffer, R. B. (1996) Psychiatric features in diffuse Lewy body disease: a clinicopathologic study using Alzheimer's disease and Parkinson's disease comparison groups. Neurology, 47, 1148-1152.

Kurita, A., Ochiai, Y., Kono, Y., et al (2003) The beneficial effect of donepezil on visual hallucinations in three patients with Parkinson's disease. Journal of Geriatric Psychiatry and Neurology, 16, 184-188.

Layton, D., Harris, S., Wilton, L. V., et al (2005) Comparison of incidence rates of cerebrovascular accidents and transient ischaemic attacks in observational cohort studies of patients prescribed risperidone, quetiapine or olanzapine in general practice in England including patients with dementia. Journal of Psychopharmacology, 19, 473-482.

Leroi, I., Brandt, J., Reich, S. G., et al (2004) Randomized placebocontrolled trial of donepezil in cognitive impairment in Parkinson's disease. International Journal of Geriatric Psychiatry, 19, 1-8.

Maidment, I. D., Fox, C. \& Boustani, M. (2006) Cholinesterase inhibitors for Parkinson's disease dementia. Cochrane Database of Systematic Reviews, issue 1. Chichester: Wiley InterScience. DOI: 10.1002/14651858.CD004747.pub2.

McKeith, I. G., Galasko, D., Kosaka, K., et al (1996) Consensus guidelines for the clinical and pathologic diagnosis of dementia with Lewy bodies (DLB): report of the consortium on DLB international workshop. Neurology, 47, 1113-1124.

McKeith, I., Del Ser, T., Spano, P., et al (2000) Efficacy of rivastigmine in dementia with Lewy bodies: a randomised, double-blind, placebo-controlled international study. Lancet, 356, 2031-2036.

Miwa, H., Morita, S., Nakanishi, I., et al (2004) Stereotyped behaviors or punding after quetiapine administration in Parkinson's disease. Parkinsonism and Related Disorders, 10, 177-180.

Morgante, L., Epifanio, A., Spina, E., et al (2004) Quetiapine and clozapine in parkinsonian patients with dopaminergic psychosis. Clinical Neuropharmacology, 27, 153-156.

Nilsson, F. M. (2004) Psychiatric and cognitive disorders in Parkinson's disease. Current Opinion in Psychiatry, 17 197-202.

Ondo, W. G., Levy, J. K., Vuong, K. D., et al (2002) Olanzapine treatment for dopaminergic-induced hallucinations. Movement Disorders, 17, 1031-1035.

Parkinson Study Group (1999) Low-dose clozapine for the treatment of drug-induced psychosis in Parkinson's disease. New England Journal of Medicine, 340, 757-763.

Parkinson Study Group (2000) Pramipexole v. levodopa as initial treatment for Parkinson disease: a randomized controlled trial. JAMA, 284, 1931-1938.

Pluck, G. C. \& Brown, R. G. (2002) Apathy in Parkinson's disease Journal of Neurology, Neurosurgery and Psychiatry, 73, 636-642.

Poewe, W. (2003) Psychosis in Parkinson's disease. Movement Disorders, 18 (suppl. 6), S80-S87.

Prueter, C., Habermeyer, B., Norra, C., et al (2003) Akathisia as a side-effect of antipsychotic treatment with quetiapine in a patient with Parkinson's disease. Movement Disorders, 18, $712-713$.

Rascol, O., Brooks, D. J., Korczyn, A. D., et al (2000) A five-year study of the incidence of dyskinesia in patients with early Parkinson's disease who were treated with ropinirole or levodopa. 056 Study Group. New England Journal of Medicine, 342, 1484-91

Rascol, O., Goetz, C., Koller, W., et al (2002) Treatment interventions for Parkinson's disease: an evidence based assessment. Lancet, 359, 1589-1598.

Samuel, M. \& Colchester, A. C. F. (2005) Structural and functional magnetic resonance imaging in neurodegenerative diseases. In Neurodegenerative Diseases (eds M. F. Beal, A. E. Lang \& A. E. Ludolph), pp. 253-289. Cambridge: Cambridge University Press.

Tandberg, E., Larsen, J. P., Aarsland, D., et al (1996) The occurrence of depression in Parkinson's disease: a community based study. Archives of Neurology, 53, 175-179.

Walker, Z., Costa, D. C., Walker, R. W., et al (2004) Striata dopamine transporter in dementia with Lewy bodies and Parkinson's disease: a comparison. Neurology, 62, 1568-1572. 


\section{MCQS}

1 Parkinson's disease dementia:

a is uncommon

b is difficult to distinguish from Lewy-body dementia

c results in deficits in executive dysfunction

d presents with hallucinations more frequently than does Alzheimer's disease

e patients are sensitive to antipsychotics.

2 As regards movement disorders in Parkinson's disease:

a medication may affect psychiatric symptoms

b they may be aggravated by psychotropic medication

c peak plasma doses of levodopa always trigger psychotic symptoms

$\mathrm{d}$ they may mask depression

e presentation together with cognitive impairment has a poor prognosis.

3 Regarding psychiatric symptoms in Parkinson's disease dementia:

a there is good evidence for antipsychotic efficacy

b depression is straightforward to diagnose

c such symptoms are uncommon

$\mathrm{d}$ they should be considered in isolation from motor symptoms

e hallucinations are most commonly tactile.
4 Cholinesterase inhibitors in Parkinson's disease dementia:

a are well tolerated

b show some efficacy for psychotic symptoms

c have established benefits for behavioural symptoms

d offer no benefit for cognitive symptoms

e assist in motor symptom control.

5 In Parkinson's disease dementia:

a hallucinations are rarer than in Parkinson's disease without dementia

b psychotic symptoms are predictive of the need for nursing home placement

c depression may be more severe than in Parkinson's disease without dementia

d MRI is useful in routine clinical practice to distinguish from Lewy-body dementia

e depression is identical to depression in people without Parkinson's disease dementia.

\begin{tabular}{|c|c|c|c|c|}
\hline \multicolumn{5}{|c|}{ MCQ answers } \\
\hline 1 & 2 & 3 & 4 & 5 \\
\hline a F & a $\mathrm{T}$ & a F & a $F$ & a $\mathrm{F}$ \\
\hline $\mathrm{b} \mathrm{T}$ & b $\mathrm{T}$ & b F & b $\mathrm{T}$ & $\mathrm{b} T$ \\
\hline c $\mathrm{T}$ & C F & C F & c F & c $\mathrm{T}$ \\
\hline $\mathrm{d} T$ & $\mathrm{~d} T$ & d F & d F & $\mathrm{d} F$ \\
\hline e $\mathrm{T}$ & e $\mathrm{T}$ & e $F$ & e $F$ & e $\mathrm{F}$ \\
\hline
\end{tabular}

